\title{
Channel Modeling for Underwater Optical Communication
}

\author{
Chadi GABRIEL ${ }^{1,2}$, Mohammad-Ali KHALIGHI ${ }^{1}$, Salah BOURENNANE ${ }^{1}$, Pierre LEON $^{2}$, Vincent RIGAUD ${ }^{2}$ \\ ${ }^{1}$ Institut Fresnel, UMR CNRS 6133, Marseille, France \\ 2 IFREMER, La Seyne Sur Mer, France \\ chadi.gabriel@ifremer.fr, \{Ali.Khalighi,Salah.Bourennane\}@ fresnel.fr, \{Pierre.Leon, Vincent.Rigaud\}@ifremer.fr
}

\begin{abstract}
We investigate in this paper channel modeling for underwater optical channels. In particular, we focus on the channel impulse response and quantify the channel time dispersion under different conditions of water type, link distance, and the transmitter/receiver parameters. Our approach is based on Monte Carlo simulations where we simulate the trajectories of emitted photons propagating in water channel towards the receiver. We show that in most practical cases, the time dispersion is negligible and does not induce any inter-symbol interference on the received symbols. The realistic channel model that we present in this paper can be used to appropriately set different system design parameters.
\end{abstract}

\section{INTRODUCTION}

The past few years have overseen a rapidly growing interest in underwater wireless sensor networks (UWSNs) which can provide knowledge about physical events happening in the volatile underwater environment. Building a sensor network adapted to aqueous environments is very delicate due to the problems such as propagation delays, limited link capacity, and energy consumption. It is well known that radio frequencies can not be used in water because they are strongly attenuated, allowing typical ranges of a few centimeters only. Use of acoustic waves is also problematic due to their limited bandwidth and very low celerity, as well as the highly energy consuming large antennas used [1], [2]. Optical underwater communication is a cost-effective and low energy consumption solution that can provide high data-rates over relatively short transmission ranges and has received a great attention since a few years. The European FP7 SENSENet project [3] considers the deployment of an UWSN in deep sea where sensor nodes communicate with each other via optical links using an adequate wavelength (in the blue/green range).

Optical communication in water is not an easy task due to high absorption and scattering undergone by the optical beam. It is of crucial importance to characterize the underwater optical channel and to set the system parameters appropriately to enable high-quality transmission. In particular, in addition to signal attenuation, scattering can create inter-symbol-interference (ISI) by causing pulse stretching. This, in turn, either degrades the quality of data transmission, especially for high data rates and over long distances [4], [5], or necessitates computationally complex signal processing at the receiver. This paper considers comprehensive modeling of the underwater optical communication link by taking different system parameters into account in view of enabling appropriate system design. We use a Monte Carlo simulation tool to simulate the trajectories of photons propagating from the transmitter towards the receiver. Different parameters such as the transmitter beam divergence, water type, link distance, and the receiver aperture size are taken into consideration, and their effect on the channel impulse response (IR) is shown. Our main result is that in most practical cases we can neglect channel dispersion due to scattering.

The reminder of paper is organized as follows. In Section II we present briefly some previous works related to underwater optical propagation modeling and specify our contributions with respect to them. In Sections III and IV we recall the main characteristics of the water channel and the main equation governing for light propagation in water. The description of our Monte Carlo simulator is provided in Section V, and in Section VI we present some numerical results to show the effect of scattering on signal transmission. Finally, Section VII concludes the paper.

\section{PREVIOUS WORKS RELATED TO UNDERWATER OPTICAL COMMUNICATION}

Several recent works have studied underwater wireless optical communication. They mostly neglect the channel dispersion and use a simple model for optical beam propagation. In [6], [7], the performance of a wireless underwater optical communication in various water types and at different ranges is studied using the simple exponential attenuation model. In [8], [9] authors study the spatial and angular effects of scattering on a laser link based on radiative transfer equation (RTE) and also present some laboratory experiments. Recently, it has been shown in [10] that channel fading due to water turbidity is negligible in most practical cases.

The two most related publications to our work are [4] and [5]. In [4], the author uses the vector RTE with the modified Stokes vector to model light scattering in water. Considering polarized light, he studies the effect of the transmission distance on channel dispersion and concludes that ISI is very limiting over long ranges $(50 \mathrm{~m})$ and for high rates $(1 \mathrm{Gbps})$. However, the water parameters considered in this work are very far from practice, as they correspond to a highly dispersive medium (that would likely apply to blood, for instance). On the other 


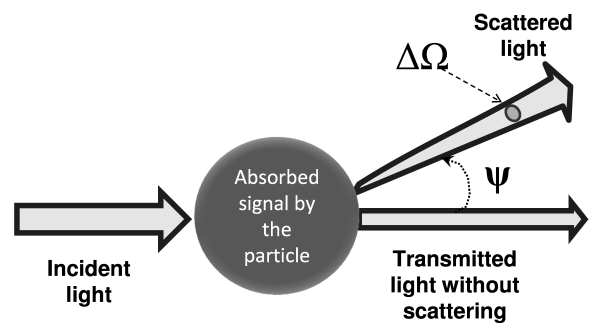

Fig. 1. Light scattering when encountering a particle in water.

hand, in [5], the authors present a laboratory experiment for a $1 \mathrm{Gbps}$ rate optical transmission system over a $2 \mathrm{~m}$ path length. They also study the temporal behavior in different water types using Monte Carlo simulations based on a simple convolutional model.

The difference of our study with respect to [4] is that here we take into account the practical system parameters, particularly concerning the transmitter and the receiver, and also consider more realistic water parameters. Also, we do not take into account light polarization because we consider intensity modulation with non-coherent detection, which is usually used in most systems due to its simplicity. Compared to [5], here we take into account the transmitter beam divergence and the receiver aperture size, and consider longer ranges (more than $10 \mathrm{~m}$ ) that are likely to be used in our system.

\section{EFFECT OF WATER ON THE OPTICAL BEAM}

The two main processes affecting light propagation in water are absorption and scattering, which both depend on wavelength $\lambda$ [11], [12]. Absorption is the irreversible loss of intensity and depends on the water's index of refraction. The spectral absorption coefficient $a(\lambda)$ is the main intrinsic optical property (IOP) to model water absorption. Scattering, on the other hand, refers to the deflection of light from the original path, which can be caused by particles of size comparable to $\lambda$ (diffraction), or by particulate matters with refraction index different from that of the water (refraction). Figure 1 illustrates scattering of the light flux when encountering a particle in water. The spectral volume scattering function (VSF) $\beta(\Psi, \lambda)$ is defined as the fraction of incident power scattered out of the beam through an angle $\Psi$ into a solid angle $\Delta \Omega$ centered on $\Psi$. The VSF is used as the main IOP to model scattering in water. Integrating the VSF over all directions, gives the scattering coefficient $b(\lambda)$ :

$$
b(\lambda)=2 \pi \int_{0}^{\pi} \beta(\Psi, \lambda) \sin (\Psi) d \Psi .
$$

The beam extinction coefficient $c$ is defined as the sum of $a$ and $b$ :

$$
c(\lambda)=a(\lambda)+b(\lambda) .
$$

Note that $a, b$, and $c$ are in units of $\mathrm{m}^{-1}$.

\section{A. Water particles}

In addition to water molecules, different particles in solution and/or in suspension in water affect absorption

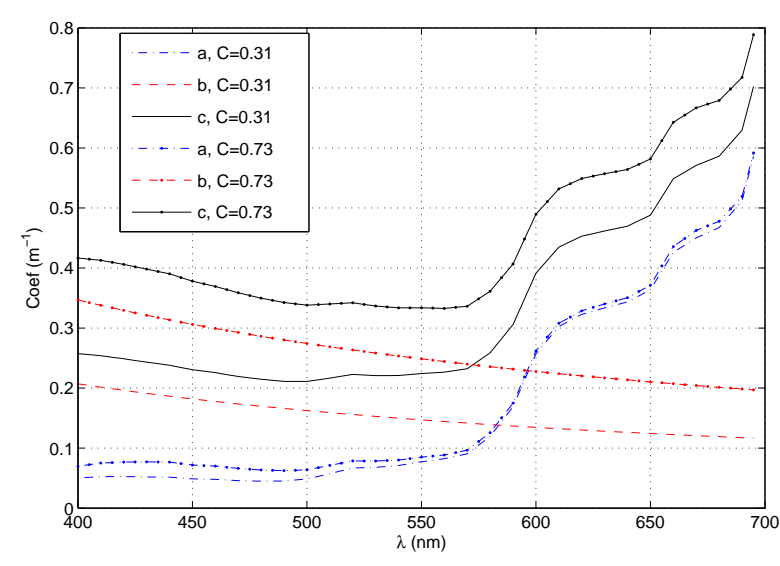

Fig. 2. Absorption, scattering, and extinction coefficients as a function of wavelength for two chlorophyll concentrations using the model of Gordon and Morel [15].

and scattering [13]. The main particles we are concerned with are: various dissolved salts, detrital and mineral components, colored dissolved organic matters, and organic matters which contribute in general to backscattering. In particular, phytoplanktons determine the optical properties of most oceanic waters because their chlorophyll and related pigments strongly absorb light in the blue and red spectral ranges. The spectral absorption and scattering coefficients $a$ and $b$ can be calculated by adding the contribution of each class of particles to the corresponding coefficients of the pure sea water. The interesting point is that one can also use the chlorophyll concentration $C$ (in $\mathrm{mg} . \mathrm{m}^{-3}$ ) as the free parameter to compute $a$ and $b$ based on bio-optical models such as those proposed by Haltrin and Kattawar [14] and Gordon and Morel [15].

We were interested to see the impact of $C$ on the absorption and scattering properties of water. We have shown in Fig. 2 curves of $a, b$, and $c$ as a function of wavelength $\lambda$ using the model of Gordon and Morel for two chlorophyll concentrations of 0.31 and $0.83 \mathrm{mg}^{-3} \mathrm{~m}^{-3}$. We notice that an increase in $C$ has negligible impact on $a$ but it affects considerably $b$.

\section{B. Water types}

Knowing that underwater matters and water quality are variant from a region to another, four major water types are usually considered [5], [9], [16]. We resume in the following different water types.

- Pure sea waters like in deep ocean: Absorption is the main limiting factor. The low $b$ and the forward angle scattering make the beam propagate approximately in a straight line.

- Clear ocean waters: There is a higher concentration of dissolved particles that affect scattering.

- Coastal ocean waters: They have a much higher concentration of planktonic matters, detritus and mineral components that affect absorption and scattering.

- Turbid harbor and estuary waters: They have a very high concentration of dissolved and in-suspension matters that make them especially constraining for 


\begin{tabular}{|c|c|c|c|c|}
\hline Water type & $C\left(\mathrm{mg} / \mathrm{m}^{3}\right)$ & $a\left(\mathrm{~m}^{-1}\right.$ & $b\left(\mathrm{~m}^{-1}\right)$ & $c\left(\mathrm{~m}^{-1}\right)$ \\
\hline \hline Pure sea & 0.005 & 0.053 & 0.003 & 0.056 \\
\hline Clear ocean & 0.31 & 0.069 & 0.08 & 0.15 \\
\hline Coastal & 0.83 & 0.088 & 0.216 & 0.305 \\
\hline Harbor & 5.9 & 0.295 & 1.875 & 2.17 \\
\hline
\end{tabular}

TABLE I

TYPICAL COEFFICIENTS FOR DIFFERENT WATER TYPES.

optical propagation.

Table I shows typical $a, b$, and $c$ parameters associated with these water types that we will consider hereafter.

\section{RADIATIVE TRANSFER THEORY}

The RTE describes the behavior of the radiance within water [13], [17]. Let us denote by $L(z, \theta, \phi, \lambda)$ the light radiance, with $z$ being the distance from the transmitter, and $\theta$ and $\phi$ the inclination and azimuthal angles, respectively. Let us define the parameter $r$ as $r=z / \cos \theta$. We have [13], [17]:

$$
\frac{d L}{d r}=-c L+L^{\mathrm{E}}+L^{\mathrm{I}} \quad\left(\mathrm{Wm}^{-3} \mathrm{sr}^{-1} \mathrm{~nm}^{-1}\right),
$$

where $L^{\mathrm{E}}$ and $L^{\mathrm{I}}$ denote path functions for elastic and inelastic scattering, respectively. Inelastic scattering, in opposition to elastic scattering, corresponds to the loss of photons due to wavelength change. Because of its relative low contribution to the general solution of the RTE, we neglect inelastic scattering in this work and do not indicate the parameter of $\lambda$ for $L$. So, integrating (3) with respect to $r$, we obtain the simplified form of RTE as follows.

$$
L(z, \theta, \phi)=L(0, \theta, \phi) e^{-c r}+L_{K}^{E},
$$

where

$$
\begin{aligned}
L_{K}^{E}= & \frac{L^{\mathrm{E}}(0, \theta, \phi) \exp (-K r \cos \theta)}{c-K \cos \theta} \\
& \times[1-\exp (-r(c-K \cos \theta))],
\end{aligned}
$$

and $K$, which is a function of $\theta$ and $\phi$, is the diffuse attenuation coefficient of radiance, and is defined as follows.

$$
K=-\frac{1}{L(z, \theta, \phi)} \frac{d L(z, \theta, \phi)}{d z} \quad\left(\mathrm{~m}^{-1}\right)
$$

Instead of solving (4) analytically, we use Monte Carlo simulation tool in this paper. Note that instead of using (4), most previous works neglect scattering and consider straight-line propagation. This comes to considering the simple Lambert's law as follows.

$$
L(z)=L(0) \exp (-c r)
$$

\section{Monte Carlo simulations}

To model the optical wave propagation in water, we use in this paper Monte Carlo simulations based on the MCML open source code [18]. The interest of this stochastic model is that besides its simplicity and flexibility, it is a rigorous approach for modeling photon transport in water. The main parameters that we take into consideration in the Monte Carlo simulator are:
- The chlorophyll concentration $C$ and the wavelength $\lambda$ to determine the coefficients $a$ and $b$ (see Subsection III-A).

- The Henyey-Greenstein parameter $g$ which is the average of the cosine of the scattering angle $\Psi$ over all scattering directions.

- The distance between the transmitter and the receiver $Z$.

- The transmitter emitted power, beam width, and maximum initial divergence angle $\theta_{0}$.

- The receiver aperture size.

The simulator relies on local probabilistic rules of photon propagation in water. Initially, each photon is launched in the medium with a unity weight. The initial position of the photon is calculated using three uniform distributions knowing the beam width and the maximum initial divergence angle. Then, the considered emitted photon travels a distance $\delta$ before interacting with a particle in the medium; what we will refer to as step size. To generate $\delta$ randomly, we consider the random variable $(\mathrm{RV}) \chi_{\delta}$ with a uniform distribution $\mathcal{U}[0,1]$ and use $\delta=-\log \left(\chi_{\delta}\right) / c$ [18]. When interacting with the particle, the photon loses a fraction of its initial weight. Lets denote the photon weight before and after interaction by $W_{\text {pre }}$ and $W_{\text {post }}$, respectively. We have [13]:

$$
W_{\text {post }}=W_{\text {pre }}(1-a / c)
$$

In addition to losing a fraction of its weight, the photon is scattered from its initial direction. To obtain the random scattering angle $\theta$, we generate a RV $\chi_{\theta}$ with the uniform distribution $\mathcal{U}[0, \pi]$, and calculate $\theta$ from $\chi_{\theta}$ according to the Henvey and Greenstein function [13]:

$$
\chi_{\theta}=\frac{1-g^{2}}{2\left(1+g^{2}-2 g \cos \theta\right)} .
$$

Lastly, we consider an azimuthal angle $\varphi$ of the scattering direction according to a uniform distribution $\mathcal{U}[0,2 \pi]$. This cycle of "step size generation $\rightarrow$ weight drop $\rightarrow$ angle scattering generation" is repeated until one of two following events happens:

- The photon weight is too small and negligible. The photon is considered as totally absorbed. This limit is set here to $10^{-4}$.

- The photon reaches the receiver. If it is in the receiver aperture, it is considered as effectively received. It is considered as lost, otherwise.

Given a number of emitted photons (related to the emitted light intensity), the accumulated weight of the photons collected at the receiver is a measure of (is proportional to) the received signal intensity. Our simulator returns the proportion of the absorbed, lost, and received photons' weights, as well as the Cartesian coordinates of the point of impact at the receiver plane. In addition, it calculates the total distance traveled by each photon until it reaches the receiver. This parameter can be interpreted as the propagation delay from the transmitter to the receiver. In our simulations, we have generated at least $10^{6}$ photons for each experiment, and have repeated the experiments at least $10^{4}$ times to obtain reliable results. 


\section{NUMERICAL RESULTS}

We provide here some simulation results to study the characteristics of the underwater optical channel. Concerning the transmitter, we consider $\lambda=532 \mathrm{~nm}$, a beam width of $3 \mathrm{~mm}$, and a maximum beam divergence of $\theta_{0}=20^{\circ}$. Also, we set the Henyey-Greenstein parameter to $g=0.924$ and consider a receiver field of view (FOV) of $180^{\circ}$. That is because in deep-sea which is our case of interest, we can effectively neglect background radiations and hence there would be no need to limit the FOV.

\section{A. Received intensity as function of distance}

Let us first see the effect of the extinction coefficient $c$ on the total received intensity $I_{r}$. We have shown in Fig. 3 curves of $I_{r}$ as a function of distance $Z$ for the four water types specified in Table I and two extreme cases of $D=5 \mathrm{~mm}$ and $50 \mathrm{~cm}$. Let us assume a tolerable loss of $-100 \mathrm{~dB}$ beyond which the signal is not detectable at the receiver (which depends on the transmitted power and the receiver sensitivity). We notice that, for $D=$ $5 \mathrm{~mm}$, the transmission range is limited to 25 and $65 \mathrm{~m}$ for clear ocean and pure sea waters, respectively, for instance. Increasing the aperture to $D=50 \mathrm{~cm}$ increases dramatically these range limits as, obviously, it allows collecting more scattered photons: Compared to $D=$ $5 \mathrm{~cm}$, the corresponding range limits increase to $50 \mathrm{~m}$ and more than $120 \mathrm{~m}$ respectively. When working in turbid harbor waters the high signal dispersion and attenuation makes communication range limited to less than a few meters.

\section{B. Channel impulse response}

The most useful information on the channel is its impulse response (IR) based on which one can specially deduce information on intensity attenuation and delay dispersion. We have conducted three sets of simulations to study the IR for different cases of link distance, receiver lens diameter, and water type. To quantify the channel time dispersion, we define the delay dispersion $\tau$ for which the IR falls $-20 \mathrm{~dB}$ below its peak.

1) IR for different water types: The IR for different water types are compared in Fig. 4 for $Z=20 \mathrm{~m}$ and $D=$ $20 \mathrm{~cm}$. The abscissa represents the absolute propagation time from the transmitter to the receiver. The case of turbid harbor waters is not represented because too few photons reach this distance for this water type, as it can be seen in Fig. 3. We notice that the channel delay dispersion $\tau$ is about $0.18 \mathrm{~ns}, 0.35 \mathrm{~ns}$, and $0.38 \mathrm{~ns}$ for pure sea, clear ocean, and coastal waters, respectively. So, for data-rates even up to $1 \mathrm{Gbps}$, channel can practically be considered as non-dispersive, and ISI as negligible.

2) Effect of receiver aperture size: The effect of receiver lens size $D$ on the IR is illustrated in Fig.5 for clear ocean water and $Z=20 \mathrm{~m}$. Obviously, use of larger lenses leads to higher photon counts: we have a $20 \mathrm{~dB}$ improvement in the IR peak by increasing $D$ from $5 \mathrm{~mm}$ to $50 \mathrm{~cm}$. Enlarging the receiver lens results also in widening the channel IR since more scattered photons are collected this way. For instance, $\tau$ is increased from $0.17 \mathrm{~ns}$ to $0.42 \mathrm{~ns}$ by increasing $D$ from $5 \mathrm{~mm}$ to $50 \mathrm{~cm}$.

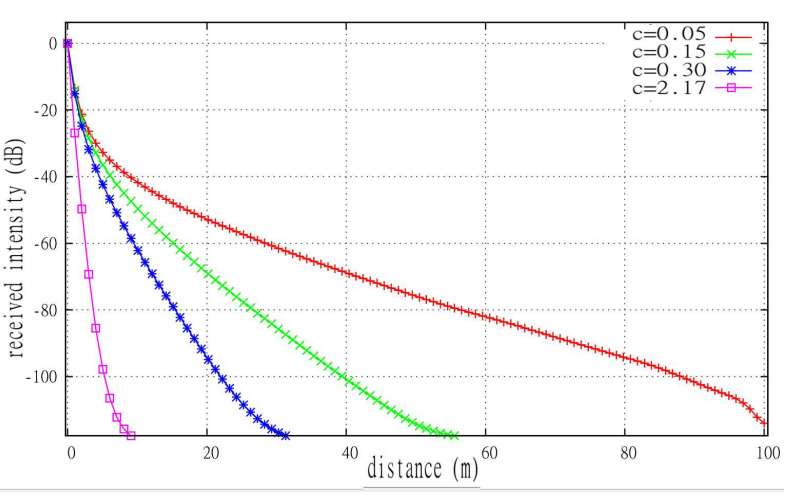

(a) $D=5 \mathrm{~mm}$

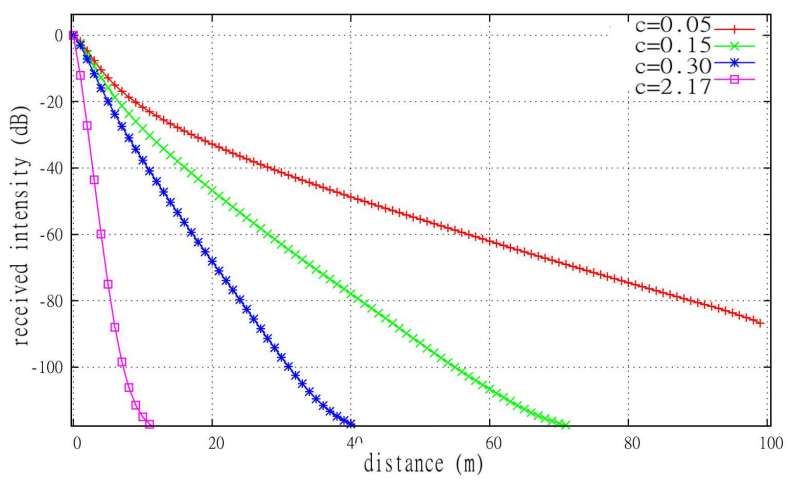

(b) $D=50 \mathrm{~cm}$

Fig. 3. Received intensity (in $\mathrm{dB}$ ) as a function of distance for different water types.

3) IR versus link distance: Figure 6 shows the channel IR for the clear ocean water with for three link distances of $Z=10,20$, and $50 \mathrm{~m}$. We have intentionally considered the extreme case of $D=50 \mathrm{~cm}$ (which could be considered as too large for a practical system) to see the impact on channel dispersion. As expected, channel dispersion increases by increasing $Z$. The channel dispersion $\tau$ is about $0.37 \mathrm{~ns}, 0.42 \mathrm{~ns}$, and $0.59 \mathrm{~ns}$ for the three $Z$ values. We see that it remains negligible even for a (relatively long) distance of $50 \mathrm{~m}$.

Lastly, Table II resumes the main results concerning the study of channel IR presented above.

\section{CONCLUSIONS}

In this article, we presented a realistic model for an underwater wireless optical channel using an elaborate Monte Carlo simulator taking into account the different transmission parameters such as the water type and the transmitter/receiver characteristics. As our main result, through this study we demonstrated that the channel delay dispersion is negligible in most practical cases. Even when working over distances up to $50 \mathrm{~m}$ in clear waters, we showed that the channel can effectively considered as frequency non-selective. As a result, we do not suffer from any ISI and do not need to perform computationally 


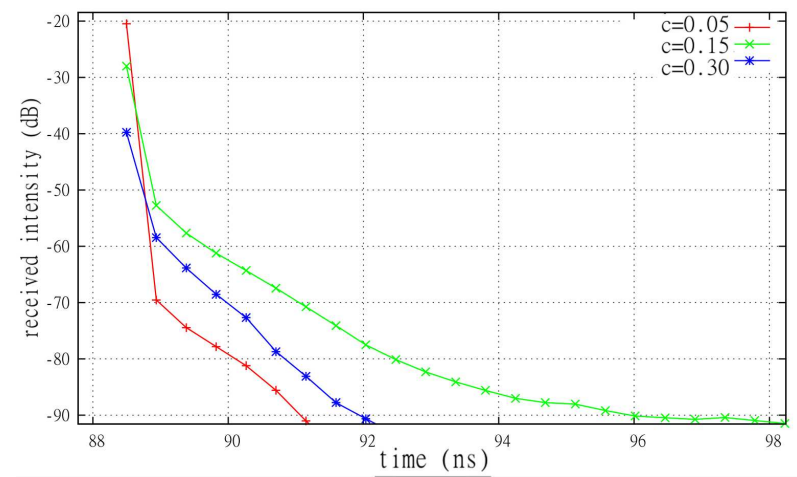

Fig. 4. Channel IR (received intensity as a function of time) for pure sea, clear ocean, and coastal waters. $Z=20 \mathrm{~m}, D=20 \mathrm{~cm}$

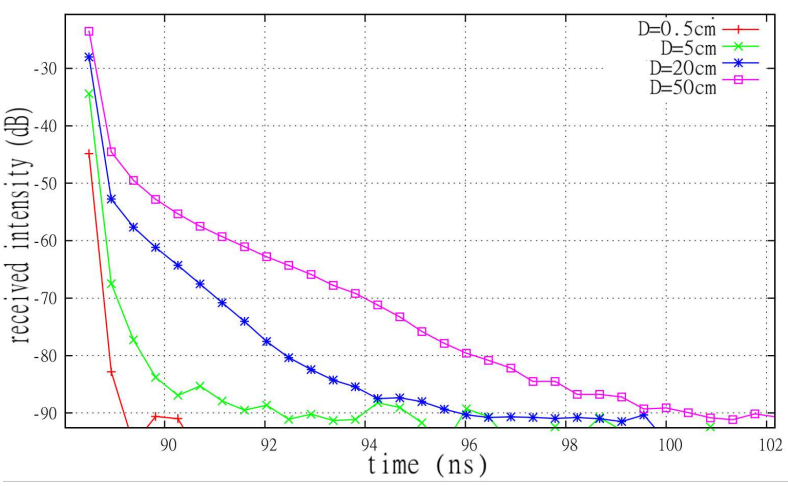

Fig. 5. Channel IR for different receiver aperature diameters $D . Z=$ $20 \mathrm{~m}$, clear ocean waters.

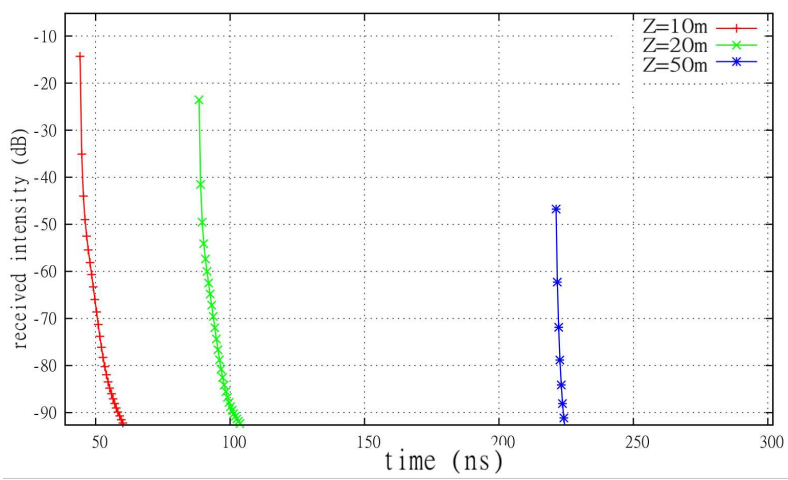

Fig. 6. Channel IR for different link distances $Z . D=50 \mathrm{~cm}$, clear ocean waters.

\begin{tabular}{|c|c|c||c|c|}
\hline$c\left(\mathrm{~m}^{-1}\right)$ & $Z(\mathrm{~m})$ & $D(\mathrm{~cm})$ & Intensity loss $(\mathrm{dB})$ & $\tau(\mathrm{ns})$ \\
\hline \hline 0.05 & 20 & 20 & -20.55 & 0.18 \\
\hline 0.15 & 20 & 20 & -28.03 & 0.35 \\
\hline 0.31 & 20 & 20 & -39.82 & 0.38 \\
\hline \hline 0.15 & 20 & 0.5 & -44.83 & 0.17 \\
\hline 0.15 & 20 & 5 & -34.43 & 0.27 \\
\hline 0.15 & 20 & 20 & -27.99 & 0.35 \\
\hline 0.15 & 20 & 50 & -23.52 & 0.42 \\
\hline \hline 0.15 & 10 & 50 & -14.29 & 0.37 \\
\hline 0.15 & 20 & 50 & -23.52 & 0.42 \\
\hline 0.15 & 50 & 50 & -46.78 & 0.59 \\
\hline
\end{tabular}

TABLE II

SUMMARY OF INTENSITY LOSS AND CHANNEL DELAY DISPERSION FOR DIFFERENT SYSTEM PARAMETERS. complex signal processing such as channel equalization at the receiver.

\section{ACKNOWLEDGMENT}

This work has been supported in part by the Marie Curie initial training network related to the FP7 SENSENet European project.

\section{REFERENCES}

[1] G. Shah, "A survey on medium access control in underwater acoustic sensor networks," in International Conference Workshops on Advanced Information Networking and Applications (WAINA), May 2009, pp. 1178-1183, Bradford, UK.

[2] F. Pignieri, F. De Rango, F. Veltri, and S. Marano, "Markovian approach to model underwater acoustic channel: Techniques comparison," in Military Communications Conference (MILCOM), Nov. 2008, pp. 1-7, San Diego, CA.

[3] International Sensor Development Network, SENSENet, http://www.eu-sensenet.net/.

[4] S. Jaruwatanadilok, "Underwater wireless optical communication channel modeling and performance evaluation using vector radiative transfer theory," IEEE Journal on Selected Areas in Communications, vol. 26, no. 9, pp. 1620-1627, Dec. 2008.

[5] F. Hanson and S. Radic, "High bandwidth underwater optical communication," Applied Optics, vol. 47, no. 2, pp. 277-283, Jan. 2008.

[6] J. H. Smart, "Underwater optical communications systems. Part 1: Variability of water optical parameters," in IEEE Military Communications Conference, Atlantic City, NJ, Oct. 2005, vol. 2, pp. 1140-1146.

[7] J. W. Giles and I. Bankman, "Underwater optical communcations systems. Part 2: Basic design considerations," in IEEE Military Communications Conference (MILCOM), Atlantic City, NJ, Oct. 2005, vol. 3, pp. 1700-1705.

[8] B. Cochenour, L. Mullen, and A. Laux, "Spatial and temporal dispersion in high bandwidth underwater laser communication links," in IEEE Military Communications Conference (MILCOM), San Diego, CA, Nov. 2008, pp. 1-7.

[9] B. M. Cochenour, L. J. Mullen, and A. E. Laux, "Characterization of the beam-spread function for underwater wireless optical communications links," IEEE Journal of Oceanic Engineering, vol. 33, no. 4, pp. 513-521, Oct. 2008.

[10] F. Hanson and M. Lasher, "Effects of underwater turbulence on laser beam propagation and coupling into single-mode optical fiber," Applied Optics, vol. 49, no. 16, pp. 3224-3230, June 2010.

[11] C.D. Mobley, B. Gentili, H. R. Gordon, Z. Jin, G. W. Kattawar, A. Morel, P. Reinersman, K. Stamnes, and R. H. Stavn, "Comparison of numerical models for computing underwater light fields," Applied Optics, vol. 32, no. 36, pp. 7484-7504, Dec. 1993.

[12] D. J. Bogucki, J. Piskozub, M.-E. Carr, and G. D. Spiers, "Monte carlo simulation of propagation of a short light beam through turbulent oceanic flow," Optics Express, vol. 15, no. 21, pp. 1398813996, Oct. 2007.

[13] C. F. Bohren and D. R. Huffman, Absorption and Scattering of Light by Small Particles, Wiley, 1988.

[14] V. L. Haltrin and G. W. Kattawar, "Self-consistent solutions to the equation of transfer with elastic and inelastic scattering in oceanic optics: I. model," Applied Optics, vol. 32, no. 27, pp. 5356-5367, 1993.

[15] C. D. Mobley, Light and Water: Radiative transfer in natural waters, Academic Press, June 1994.

[16] G. Kervern, "Lidars sous-marins," Techniques de l'Ingénieur, vol. 6, no. E4325, pp. 1-7, Nov. 1997.

[17] A. Ishimaru, Wave Propagation and Scattering in Random Media, IEEE Press, 1997.

[18] L. Wang, S. L. Jacques, and L. Zheng, "MCML, Monte Carlo modeling of light transport in multi-layered tissues," Tech. Rep., Laser Biology Research Laboratory, University of Texas, M.D. Anderson Cancer Center, Nov. 1995. 